\title{
La acreditación de programas de educación superior
}

\section{The accreditation of higher education programs}

\author{
MORALES, Betzabeth Dafne†*, PÉREZ-SÁNCHEZ, Mónica, MEJÍA-ROCHA, Mónica Isabel \\ Universidad de Guanajuato, Departamento de Gestión y Dirección de Empresas. Lascurain de Retana No. 5; Ciudad de \\ Guanajuato, Guanajuato, México.
}

ID $1^{\text {er }}$ Autor: Betzabeth Dafne, Morales / ORC ID: 000-0001-9176-6391, Researcher ID Thomson: X-2395-2018, CVU CONACYT ID: 952932

\author{
ID $1^{\text {er }}$ Coautor: Mónica, Pérez-Sánchez / ORC ID: 0000-0002-1327-2174, Researcher ID Thomson: AAA-1697-2019, CVU \\ CONACYT ID: 563029
}

ID $2^{\text {do }}$ Coautor: Mónica Isabel, Mejía-Rocha / ORC ID: 0000-0003-01497445, CVU CONACYT ID: 609586

DOI: $10.35429 / J U P .2019 .9 .3 .1 .10$

Recibido 10 Julio, 2019; Aceptado 28 Septiembre, 2019

\section{Resumen}

La necesidad de acreditar los programas educativos, surge en la década de los 80, cuando se comenzó a dar importancia a los procesos de evaluación, reconociendo y garantizando la calidad en la educación. Organismos internacionales como la Organización de las Naciones Unidas para la Educación, la Ciencia y la Cultura (UNESCO) y la Organización para la Cooperación y Desarrollo Económicos (OCDE) han sido impulsores de las acreditaciones desde su comienzo, creando políticas y programas en conjunto de los gobiernos del mundo. El objetivo de esta investigación consisitió en analizar las acreditaciones con las que cuentan los diferentes programas de educación superior de una Universidad Pública y el impacto que generan en la calidad de los mismos. Se utilizó una metodología cualitativa, aplicando entrevistas semiestructuradas a los coordinadores académicos. Dentro de los principales resultados, se muestra la relevancia de generar estrategias para mejorar la eficiencia terminal y por ello se ha puesto especial énfasis en las acciones de tutoría y acompañamiento al alumno.

\begin{abstract}
The need to accredit educational programs arises in the 1980 s, when evaluation processes began to be given importance, recognizing and guaranteeing quality in education. International organizations such as the United Nations Educational, Scientific and Cultural Organization (UNESCO) and the Organization for Economic Cooperation and Development (OECD) have been promoters of accreditations since its inception, creating policies and programs in set of world governments. The objective of this research was to analyze the accreditations that the different higher education programs of the University of Guanajuato have and the impact they generate on their quality. A qualitative methodology was used, applying semi-structured interviews to academic coordinators. Among the main results, there is the relevance of generating strategies to improve terminal efficiency and for this reason, special emphasis has been placed on tutoring and student support
\end{abstract}

Citación: MORALES, Betzabeth Dafne, PÉREZ SÁNCHEZ, Mónica, MEJÍA ROCHA, Mónica Isabel. La acreditación de programas de educación superior. Revista de Políticas Universitarias. 2019. 3-9: 1-10.

\footnotetext{
* Correspondencia del Autor (Correo electrónico: dafne @ugto.mx)

$\dagger$ Investigador contribuyendo como primer autor.
} 


\section{Introducción}

Los programas de estudio de educación superior ofertados por diversas instituciones, tienen entre sus importantes características, el hecho de contar con una planificación estructurada que debe cumplir con determinados estándares de calidad, los cuales son evaluados por organismos responsables de verficar que cumplan con las características holísticas para brindar un programa de calidad. Lo anterior hace referencia, no solo al contenido temático de las unidades de aprendizaje, sino también a las instalaciones universitarias, elementos tecnológicos, formación y actualización de la plantilla docente, posibilidades de estancias o intercambios académicos, entre otros.

El objetivo de la presente investigación es analizar el impacto de las acreditaciones en los programas de estudio de una univesidad pública, describiendo de forma cualitativa, la perspectiva de los actores involucrados directamente, es decir, los coordinadores académicos, a través de una entrevista semi estructurada. De igual forma, cuantitativamente se realizó una encuesta para identificar el grado de conocimiento que tienen los estudiantes sobre las acreditaciones de sus programas de estudio, pero para fines de este artículo, se presentan unicamente los resultados de la parte cualitativa.

En los siguientes apartados se hablará sobre el contexto de las acreditaciones y los principales organismos que participan en dichos procesos a nivel internacional y nacional, iniciando por una definición del término central de esta investigación. Posteriormente se realiza una revisión de literatura sobre estudios relacionados con el tema, con la finalidad de lograr identificar aspectos semejantes y otros que pueden complementar el estudio de la presente investigación.

De igual forma, se analiza el caso específico de una de las divisiones de la universidad pública, identificando el proceso a seguir para las acreditaciones tanto nacionales como internacionales y cuál es el impacto, tanto de la parte administrativa y de gestión, como de la parte académica.
En un cuarto apartado se habla de la metodología utilizada para recabar la información, para posteriormente mostrar los resultados obtenidos de las entrevistas a diferentes coordinadores de distitntas divisiones, y cerrando con un apartado de conclusiones, en las que se analizan las características tangibles e intangibles de los diversos procesos.

\section{Desarrollo}

\section{Contexto de la investigación}

El Consejo para la Acreditación de la Educación Superior A.C, señala que una la acreditación es "el reconocimiento formal y público otorgado por un ente externo a un programa académico que ha logrado avances significativos en el cumplimiento de su misión y objetivos declarados, y satisface así un conjunto acordado de criterios, indicadores y estándares de pertinencia y calidad establecidos por un Organismo Acreditador". Entonces bien, las acreditaciones son otorgadas por cuerpos acreditadores, organizaciones privadas y no gubernamentales creadas específicamente para calificar programas e instituciones con alta calidad educativa.

Las acreditaciones académicas surgieron en la década de los 80, al dar importancia a los procesos de evaluación, y funcionan para reconocer y garantizar la calidad en la educación. Organismos internacionales como la Organización de las Naciones Unidas para la Educación, la Ciencia y la Cultura (UNESCO) y la Organización para la Cooperación y Desarrollo Económicos (OCDE) han sido promotores e impulsores de las acreditaciones desde su comienzo, creando políticas y programas en conjunto de los gobiernos del mundo.

Las acreditaciones académicas se dividen y categorizan de acuerdo con diferentes criterios. Dentro de estos podemos encontrar por región, grado académico, área de estudios, institución, entre otras. Existen consejos internacionales y nacionales que funcionan para regular $\mathrm{y}$ supervisar a los organismos acreditadores que a su vez califican la calidad de los programas educativos. 
Estos consejos concentran aquellos organismos, instituciones y programas reconocidos por ellos en una base de datos formando un catálogo. Cabe destacar que todos los organismos y acreditaciones son independientes, los consejos solo funcionan como moderadores.

Los consejos pueden a su vez estar afiliados a otras redes de colaboración internacionales, un ejemplo es The International Network for Quality Assurance Agencies in Higher Education (INQAAHE) una asociación internacional formada por casi 300 organismos activos en teoría y práctica para garantizar la calidad de los estudios superiores. La mayoría de los miembros por los que está conformada esta red son consejos que operan de diferentes formas, aunque también aceptan organizaciones que tienen interés en garantizar la calidad de la educación.

En México, se encuentra el Consejo para la Acreditación de la Educación Superior, A.C. (COPAES) y es un organismo autorizado por la Secretaría de Educación Pública para reconocer formalmente y regular a aquellos organismos acreditadores en México. Tiene su origen gracias a que la Asociación Nacional de Universidades e Instituciones de Educación Superior (ANUIES) en el año 2000 llego al acuerdo de formar dicho organismo.

La COPAES funciona como el órgano mediador de las acreditaciones en México, teniendo contacto de igual forma con organismos acreditadores y las instituciones que desean obtener una. Por un lado, supervisa que los organismos estén teniendo un desempeño adecuado y del otro brinda asesorías, además de difundir e informar sobre los programas e instituciones de calidad. Según el directorio de la COPAES hay 3962 programas registrados, pero solo 3411 se encuentran vigentes de 30 organismos acreditadores en 368 instituciones de educación media superior. En el Estado de Guanajuato hay en total 133 programas vigentes en 20 instituciones, en total hay 24 organismos acreditadores en el Estado.
Los 5 organismos con mayor número de programas acreditados en México son el Consejo de la Acreditación de la Enseñanza de la Ingeniería A.C. (CACEI) 1003 programas en México y 30 en el estado, el Consejo de la Acreditación en Ciencia Administrativas Contables y Afines A.C. (CACECA) con 755 programas y 33 en Guanajuato, el consejo Nacional de Acreditación en Informática y computación A.C. (CONAIC) 166 programas, 9 en Guanajuato, el Comité Mexicano de la Acreditación de la Educación Agronómica A.C. (COMEAA) 126 programas, 7 en el estado, y el quinto organismo es la Asociación para la Acreditación y Certificación en Ciencias Sociales A.C. (ACCECISO) con 118 programas aunque solamente uno en Guanajuato.

\begin{tabular}{|l|l|l|}
\multicolumn{4}{|c|}{ Organismos yéxico } & \multicolumn{2}{c|}{ Guanajuato } \\
\hline CACEI & 1003 & 30 \\
\hline CACECA & 755 & 33 \\
\hline CONAIC & 166 & 9 \\
\hline COMEAA & 126 & 7 \\
\hline ACCECISO & 118 & 1 \\
\hline CNEIP & 108 & 3 \\
\hline COAPEHUM & 107 & 4 \\
\hline ANADEPH & 105 & 4 \\
\hline CONAET & 105 & 7 \\
\hline COMAEM & 80 & 3 \\
\hline COMPAPROD & 80 & 8 \\
\hline CAESA & 70 & 2 \\
\hline COFEDE & 59 & 3 \\
\hline CONAED & 58 & 1 \\
\hline CEPPE & 52 & - \\
\hline CONACE & 51 & 2 \\
\hline CACEB & 49 & 1 \\
\hline CONAC & 49 & 2 \\
\hline COMACE & 45 & 3 \\
\hline CONAECQ & 38 & - \\
\hline CONACAPREN & 37 & 2 \\
\hline CONAEDO & 35 & 2 \\
\hline COMACEF & 29 & 1 \\
\hline CONEVET & 18 & - \\
\hline ANPROMAR & 14 & - \\
\hline CONACI A.C. & 14 & 2 \\
\hline CAPEM & 13 & 1 \\
\hline COMACAF & 13 & - \\
\hline CAPEF & 12 & 2 \\
\hline COMACEO & 2 & - \\
\hline TOTAL & & \\
\hline
\end{tabular}

Tabla 1 Organismos acreditadores y número de programas acreditados

Fuente: Peña, 2019 


\section{Revisión de Literatura}

Silas (2014) en su artículo calidad y acreditación en la educación superior: retos y realidades para América Latina, hace referencia a la Declaración Mundial sobre la Educación Superior del siglo XXI (UNESCO) donde se estableció que la calidad en la educación superior debe comprender: enseñanzas y programas académicos, investigación y becas, personal, estudiantes, instalaciones, y servicios a la comunidad $y$ al mundo universitario. La UNESCO señala que educación terciaria se determina en función de los insumos con lo que se cuente y la forma en la que interactúan entre sí, dando respuesta a una educación de calidad. De esta manera, la UNESCO propone:

- Adecuada selección de académicos.

- Actualización constante de los académicos.

- Movilidad estudiantil.

- Uso de TIC's.

En el proceso de evaluación participa personal de enseñanza e investigación, estudiantes y administrativos, por ellos es muy importante que exista comunicación estratégica y asertiva entre las diversas áreas (Silas, 2014).

Por otro lado, Casas y Olivas (2011) en su investigación "El proceso de acreditación en programas de Educación Superior: un estudio de caso" mencionan que la Red Iberoamericana para la Acreditación de la Calidad de la Educación Superior (RIACES) habla del concepto de calidad como el "Grado en el que un conjunto de rasgos diferenciadores inherentes a la educación superior cumple con una necesidad o expectativa establecida. En una definición laxa se refiere al funcionamiento ejemplar de una institución de educación superior. Propiedad de una institución o programa que cumple los estándares previamente establecidos por una agencia u organismo de acreditación" (RIACES, citado en Casas y Olivas, 2011). Salas (2013) cita a González y Torre, quienes señalan que la calidad en la educación superior puede evaluarse mediante indicadores, criterios y variables que permitan garantizar a la sociedad la pertinencia de los saberes ofertados por las Instituciones de Educación Superior, las cuales se concentran tradicionalmente en:
- Planes y programas de estudio.

- Plantilla docente.

- Alumnos.

- Infraestructura.

- Financiamiento.

- Procesos académico-administrativos.

De igual manera, es importante analizar más a fondo el concepto de acreditación, puesto que es el tema central de la investigación, y que es parte fundamental para el análisis de la metodología. Según el Diccionario de la Real Academia Española acreditar significa "hacer digna de crédito alguna cosa, probar su certeza o realidad; afamar, dar crédito o reputación, dar seguridad de que alguna persona o cosa es lo que representa o parece"

Silas (2014) indica que la acreditación es la capacidad técnica de una instancia u organización para lograr que sus actividades correspondan de conformidad con lo esperado, es decir, trata el reconocimiento que otorga un organismo acreditador, que puede ser gubernamental o no (aunque lo usual es que no lo sea), autorizado y reconocido, que señala formalmente que un programa educativo de una institución educativa ha mostrado suficiencia en:

- Estructura.

- Organización.

- Funcionamiento.

- Insumos.

- Procesos de enseñanza.

- Servicios.

- Pertinencia social y professional.

- Resultados educativos. 
Por su parte, Audina (2003) plantea cuatro ámbitos en los que puede analizarse la problemática de la dimensión internacional de las acreditaciones que desde un punto de vista científico pueden resultar como parte importante en el análisis del porque la mayoría de los programas de educación superior solo tienen acreditaciones nacionales y no internacionales. Los cuatro ámbitos corresponden a:

a) ¿quién evalúa y acredita?

b) ¿Qué se evalúa y acredita?

c) ¿cómo se evalúa y acredita?

d) ¿cuáles son los productos de la evaluación y acreditación?

El primer ámbito lo constituyen las organizaciones encargadas de estas funciones. El segundo incluye los objetos de la evaluación y acreditación. El tercero comprende los procesos y procedimientos de las evaluaciones y el cuarto se relaciona con la naturaleza e impactos de los productos de las evaluaciones y acreditaciones (Audina, 2003).

Es indispensable dejar en claro que acreditación y evaluación, aunque tiene variables similares no son lo mismo, pues muchas veces suelen confundirse con estos términos ya que ambos aseguran un cierto grado de calidad, aunque no en la misma medida, sin embargo, resaltamos la importancia de ambos en esta investigación Egido y Haug citan a Vroeijenstijn (2003) quien nos dicen que, aunque tienen aspectos comunes, evaluación y acreditación no son lo mismo. La acreditación hace referencia a una declaración formal, independiente, sobre si se cumplen o no determinados requisitos. Mientras la evaluación puede ser interna, la acreditación tiene que ser externa.

Este carácter externo de la acreditación tiene como fundamento el hecho de que los procesos internos de evaluación y de búsqueda de calidad, aunque necesarios, se muestran insuficientes. La calidad debe ser demostrada y ello requiere algún tipo de certificación externa, especialmente si se pretende alcanzar la comparabilidad o el reconocimiento público (Egido Gálvez, I. y Haug, G. 2006).

\section{Educación Superior en México}

Para hablar un poco de los antecedentes históricos que ha tenido la educación superior en México, tomaremos algunos párrafos de un artículo científico titulado "La Educación Superior en México, Tendencias y Desafíos" por parte de las investigadoras Cruz López, Y. y Cruz López, A. K. (2008). México tiene una larga tradición e historia en educación superior, la universidad fue una de las primeras instituciones que se creó después de la conquista. Fundada en 1551, la Real y Pontificia Universidad de México, se convertiría en la Universidad Nacional Autónoma de México (UNAM). En la segunda mitad del Siglo XX se experimentó un crecimiento en el ámbito de la educación superior, tanto en el tipo de instituciones como en el número de estudiantes, profesores y áreas de investigación. En la actualidad, la educación superior se concibe como un instrumento vital para la modernización de México. El Programa Nacional de Educación 2001-2006 ofrece una visión de la educación superior al año 2025:

- La educación superior será la palanca impulsora del desarrollo social, de la democracia y la convivencia multicultural. Proporcionará a los mexicanos los elementos para su desarrollo integral y formará científicos, humanistas y profesionales, en todas las áreas del saber, portadores de conocimientos de vanguardia y comprometidos con las necesidades del país.

- Para el primer cuarto del siglo, el sistema de educación superior estará conformado por 32 sistemas estatales, contará con un amplio respaldo por parte de la sociedad y atenderá a más de la mitad de la población entre 19 y 23 años con una oferta amplia, flexible y diversificada de programas educativos en instituciones de diversos perfiles tipológicos.

- La sociedad estará plenamente informada del desempeño académico y del uso de los recursos de todas las instituciones de educación superior, con sustento en procesos consolidados de evaluación y acreditación. Para construir esta visión se necesita el compromiso de las instituciones involucradas, la participación entusiasta de las comunidades educativas y el apoyo de las autoridades federal y estatales. No es una tarea fácil e implica grandes y complejos.

MORALES, Betzabeth Dafne, PÉREZ SÁNCHEZ, Mónica, MEJÍA ROCHA, Mónica Isabel. La acreditación de programas de educación superior. Revista de Políticas Universitarias. 2019 


\section{Caso Universidad Pública}

Ahora bien, la División a estudiar, cuenta con las siguientes Acreditaciones a nivel Nacional por el Consejo de Acreditación en la Enseñanza de la Contaduría y Administración (CACECA), en las licenciaturas de Administración de la Calidad y la Productividad, Comercio Internacional, Contador Público, Relaciones Industriales y Sistemas de Información Administrativa. Asimismo, por el Consejo Nacional para la Calidad de la Educación Turística A.C. (CONAET) en la Licenciatura de Administración de Recursos Turísticos.

De la misma manera por el Consejo Nacional de Acreditación de la Ciencia Económica (CONACE) en la Licenciatura en Economía. Por otro lado, cuenta con las siguientes Acreditaciones a Nivel Internacional por el Consejo de Acreditación de Ciencias Sociales, Contables y Administrativas en la Educación Superior de Latinoamérica A.C. (CACSLA) en las licenciaturas de Administración de la Calidad y la Productividad y Comercio Internacional. Igualmente, por el Sistema de Certificación UNWTO TedQual en la Licenciatura de Administración de Recursos Turísticos. Además, en los programas de posgrado, están acreditadas dos Maestrías por los Comités Interinstitucionales para la Educación Superior (CIEES) y uno más se encuentra en el Programa Nacional de Posgrados de Calidad del CONACYT.

Asimismo, muy apegado a la revisión de literatura, los organismos acreditadores evalúan, aspectos como:
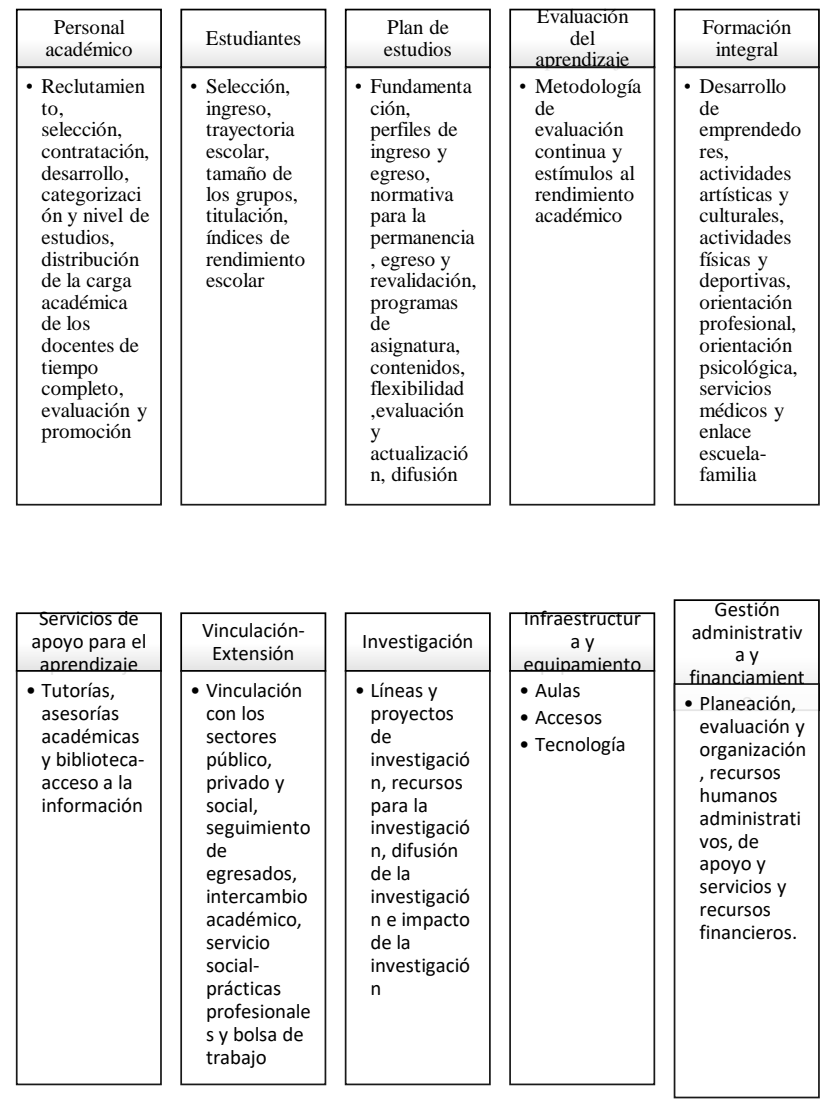

Figura 1

\section{Metodología a desarrollar}

La metodología utilizada para recabar información acerca de los programas de estudio de licenciaturas y posgrados de la universidad de Guanajuato que contaran con alguna acreditación se realizó a través de una entrevista a los coordinadores de las licenciaturas y posgrados de uno de los Campus de la Universidad Pública, que contaran con una acreditación. La entrevista se realizó con el fin de conocer desde un punto de vista profesional, las motivaciones y esfuerzos que realizaron los coordinadores en el momento de gestionar el proceso de la acreditación, además de recolectar información más detallada del beneficio para los estudiantes y el impacto de la calidad en los programas de estudio.

Fue una entrevista en la que se pretendía conocer la mayor parte de el proceso de acreditación, así como los principales obstáculos y observaciones que se presentan a la hora de recibir una acreditación o reacreditación. 
Dentro de las preguntas de la entrevista semi estrucutrada, se encontraban el grado y el nombre tanto del programa de estudio como del coordinador para posteriormente comenzar con el nombre y el organismo que habían acreditado al programa de estudio pues a partir de aquí cada uno podría decirnos como fue su experiencia con la acreditación que actualmente tenían. El tiempo del proceso, los actores involucrados, los aspectos que suelen mantenerse como fortalezas y aquellas áreas de oportunidad que no han podido solventarse, conociendo las razones.

\section{Resultados}

Se realizaron un total de 22 entrevistas en las 6 divisiones del campus. Para definir lo que es una acreditación los coordinadores utilizaron palabras como reconocimiento, organismo acreditador, proceso, programa de estudios, estándares de calidad, garantizar, calidad educativa y alumnos. De ello, se puede conformar la definición como: la acreditación es el reconocimiento al programa de estudios por un organismo acreditador, mediante un proceso de evaluación que establece estándares de calidad con el fin de garantizar la calidad educativa para formar alumnos competentes en el campo laboral.

La motivación principal para obtener una acreditación son los estudiantes, ya sea para dar confianza a los que desean estudiar estos programas o los que ya lo estudian tengan acceso a una educación de mayor calidad, así como la obtención de becas y apoyos. Otra de las razones para realizar el proceso de acreditación es saber el nivel en el que se encuentra el programa y las debilidades para mejorar en el futuro, es decir utilizarlo como herramienta de mejora continua. En el área de ingenierías, el organismo encardado de las acreditaciones es el Consejo de Acreditación de la Enseñanza de la Ingeneria (CACEI) mientras que organismo responsable de la acreditación del doctorado es el Consejo Nacional de Ciencia y Tecnología (CONACYT). Uno de los coordinadores resume el proceso en 6 etapas: la primera es capacitarse para aprender la forma correcta de llenado de los formularios necesarios, la segunda es la recopilación de la información necesaria, la tercera reunir las evidencias solicitadas, la cuarta etapa es gestionar una visita de los evaluadores, la quinta atenderlos para presentar las evidencias, y la sexta, se espera el dictamen de la evaluación.
Los coordinadores coinciden en que los aspectos a evaluar por parte del organismo acreditador son: personal académico, estudiantes, plan de estudios, valoración y mejora continua, infraestructura y equipamiento y por último soporte institucional. En cuanto a los principales obstáculos para llevar el proceso de acreditación señalan que lo más difícil es la participación de todos, debido a que es un proceso de obtención de información requiere de muchas actividades que se deben realizar y documentar en conjunto, pero desgraciadamente todos los involucrados tienen muchas otras responsabilidades y es complicado encontrar momentos donde puedan coincidir todos. Otro de los obstáculos podría ser no reunir los requisitos en el tiempo que se establece y no alcanzar el nivel esperado. Otras de las debilidades que mencionan los coordinadores son la desorganización, el rezago estudiantil, los índices de reprobación, niveles bajos de titulación y fortalecer un programa efectivo de difusión del programa y programas de vinculación.

Las principales fortalezas que se identifican en los programas son docentes de calidad, la alta productividad de los estudiantes, los objetivos educacionales, las áreas de oportunidad laboral, algunos consideran que las instalaciones son una fortaleza de los programas, pero en relación a la cantidad de alumnos que han venido admitiendo en los últimos años ha causado un sobrecupo, preponderadamente en aquellos programas en los que realizan actividades en laboratorios específicos, por lo que ahora es un foco rojo y una debilidad que pone en amenaza la calidad de los programas educativos.

Los coordinadores también comentaron una lista de las principales observaciones de los programas, la principal fue el seguimiento de los egresados, como inserción laboral, así como mejorar la trayectoria de los alumnos para evitar el rezago, disminuir el número de reprobados y bajas terminales. Una minoría mencionó que una de las observaciones que les hacen es aumentar la matrícula de alumnos y mejorar la infraestructura. 
Todos consideran que tiene un gran impacto que las carreras estén acreditadas y lo relacionan con el acceso a mayores convocatorias y apoyos por parte de los alumnos dando prestigio a la universidad y permitiendo contar con recurso para la mejora continua. Los beneficios que consideran para los estudiantes son facilidades de beca, el reconocimiento en otros países, simplemente saber que el programa que cursan cumple con la calidad y los estándares esperados. La mayoría de los coordinadores concuerdan en que los jóvenes que desean ingresar a una carrera en la universidad deben saber cuáles programas están acreditados porque les permite tomar una mejor decisión.

En el área de Sociales y Humanidades tienen una acreditación por parte del Consejo para la Acreditación de Programas Educativos en Humanidades (COAPEHUM) y consideran que las acreditaciones de un programa de estudio son procesos de evaluación en busca de la calidad y que su principal objetivo son los estudiantes, profesores, plan de estudios, investigación y publicación. Sobre el proceso de esta acreditación los pasos principales son muy similares a lo que comentaron en la división pasada:

\section{1.- Realizar la solicitud al organismo}

2.- El organismo proporciona instrumentos de evaluación y capacitación

\section{3.- El programa reúne la documentación}

4.- Se entrega la documentación al organismo y hacen observaciones

5.- Se corrigen las observaciones y envían evaluadores para verificar in situ

6.- Los evaluadores emiten un dictamen y entregan la acreditación a la Universidad.

En cuanto a las principales observaciones se encuentra que la infraestructura es la principal debilidad y el bajo índice de titulación. Como fortaleza se considera el cuerpo docente y los maestros son expertos en la materia.
El impacto y los beneficios que tienen las acreditaciones en la calidad de los programas de estudios, es observar qué es en lo que pueden y deben mejorar, fijando su atención en ello para después actuar, aunque generalmente los impactos positivos son a largo plazo. Es importante dar a conocer a los aspirantes las acreditaciones de los programas de estudio y sobretodo que conozcan su significado, comprendiendo que son reconocimientos por parte de alguna institución especializada donde manifiesta que la carrera cumple con lo necesario para ser de calidad por lo que es una iniciativa de la universidad ya que busca ofrecer calidad a los estudiantes y quiere que los programas, maestros e instalaciones cumplan con los requerimientos de calidad.

Es decir, las acreditaciones, son evaluaciones de calidad realizadas por organismos externos y que la mayoría de las veces las razones para obtenerlas son por el cumplimiento de las políticas institucionales en busca de la mejora continua. Uno de los obstáculos transversales que se mencionaron en las entrevistas es que los profesores de tiempo completo tienen una carga de trabajo que puede llegar a ser abrumadora y se vuelve un reto dedicar tiempo a reunir la gran cantidad de evidencias e información necesarias para el proceso.

Uno de los mayores impactos de las acreditaciones es la actualización de los planes de estudios, ya que en los últimos años las acreditaciones se han convertido casi en una obligación para los programas, lo cual mantiene una cultura de la autoevaluación constante y, algunos casos, procura mejoras en los programas logrando que los estudiantes tengan la certeza de que se cumplen con los parámetros de calidad, aunque, también comentaba otro coordinador, que para él, no es necesario que los alumnos que deseen ingresar a alguna de las carrera de la UG tengan conocimiento de las acreditaciones ya que muchas veces no es factor decisivo para elegir una carrera.

Como bien lo señaló una de las entrevistadas, las acreditaciones consisten en una evaluación acerca del funcionamiento y resultados de un programa académico, bajo un conjunto de criterios que buscan certificar que los distintos procesos académicos y administrativos se realicen de manera eficiente. 
Implica la presentación de indicadores medibles y evidencia documental, que respalde la información cualitativa y cuantitativa de un programa. Asimismo, se integran metas, con la finalidad de dar seguimiento al programa y de introducir las mejoras necesarias, en función del dictamen producto del proceso de acreditación.

Una acreditación importante es la del Padrón Nacional de Posgrados de Calidad de CONACYT, en el cual se tiene además de los anteriores impactos y beneficios, que los estudiantes, al ser de dedicación exclusiva, reciben una beca que es asimilable a un sueldo promedio estándar. La acreditación la otorga Conacyt y tiene una similitud muy parecida al proceso que han descrito los coordinadores de las licenciaturas, pues la acreditación se realiza con base en una Convocatoria emitida por Conacyt, en la que se especifican los aspectos del programa que serán evaluados. Para cumplir con lo anterior se tiene que conjuntar una serie de instrumentos de medición de metas y procedimientos académicos del programa, así como de los productos obtenidos: graduados, tesis, producción académica de los profesores que participan en el programa, principalmente. Todo lo anterior es revisado por un comité, integrado por académicos del área al que pertenece el programa, quienes emiten un dictamen sobre la información presentada, que incluye recomendaciones para el mejoramiento futuro del programa.

Otra de las observaciones recurrentes es la necesidad de incrementar el número de profesores insertos en el Sistema Nacional de Investigadores (SNI), así como cumplir con la eficiencia terminal, es decir, la culminación de las tesis en el plazo indicado por CONACYT. Con respecto a las fortalezas, nuevamente surge la planta de profesores, con una experiencia amplia y una producción de gran valor académico, así como su valiosa calidad docente. Asimismo, con el tiempo se han desarrollado una serie de instrumentos de medición de las metas del programa, que permiten identificar los logros $\mathrm{y}$ problemas inherentes al funcionamiento académico del mismo. Finalmente, los procesos de toma de decisiones se han depurado mediante la distribución de las responsabilidades a través de comités, que permiten un seguimiento especializado de las operaciones del programa.
El proceso de acreditación por parte de CONACYT para los posgrados es muy similar a las de licenciatura, pero más a detalle el proceso comienza con unos ocho meses de anticipación antes de que expire la acreditación y se tiene que reunir la evidencia que comprueba que los profesores publican, que los alumnos publican, que hay convenios con otras universidades, etc y también la parte institucional, si existen políticas internas para garantizar los procesos tienes que dar evidencia de que en la pagina de internet de la universidad están esos documento, después de generar esta información la envías a CONACYT, y luego de manera ciega seleccionan a tres coordinadores de posgrados similares para que vayan a comprobar todo lo que esta en los documentos que previamente enviaste, además de realizar observaciones al todo lo relacionado con el programa, para esto las evaluaciones por parte de los coordinadores que eligió CONACYT deben ser sin previo aviso $y$ sin que el programa este enterado de que en ese momento hay un evaluador con ellos, posteriormente los evaluadores emiten un dictamen de si se cumple o no con los criterios para recibir la acreditación nuevamente.

\section{Conclusiones}

El proceso de acreditación depende de cada organismo acreditador en el cual hay que entregar determinadas evidencias y cumplir con los indicadores que en este caso CACECA, CACSLA, CONAET, TedQual, CONACE, CIEES y PNPEC, determinan. Sin embargo, las coordinaciones pueden enfrentarse a obstáculos para el proceso, algunas ocasiones los lineamientos o indicadores no se apegan al programa, la metodología y el tiempo para reunir las evidencias, o se cuenta con poco apoyo de las diferentes áreas y los sistemas administrativos. Por tanto, dentro de las principales observaciones mas comunes que hacen a los programas de estudio, es el nivel de inglés, la cantidad de titulados, las prácticas, la deserción de alumnos, el trabajo colegiado de los profesores, la movilidad nacional e internacional y la vinculación de los estudiantes hacia el sector laboral.

Las expectativas se han cumplido, ya que obtienen las acreditaciones, además de las reacreditaciones y el trabajo constante para no solamente obtener acreditaciones a nivel nacional, sino también de manera internacional. 
Por tanto, el impacto que tienen las acreditaciones en calidad de los programas de estudio, se ve reflejado en el proceso de enseñanza-aprendizaje, en la calidad del programa, en la certeza y la confianza de formar personas competitivas. Además, que los alumnos son beneficiados, aunque no se vea de una manera directa, pues al estar acreditado deben cumplir con indicadores, como en áreas para prácticas, bibliotecas, salas de cómputo, espacios y convenios para prácticas, profesores, instalaciones, servicios que no se ven de manera directa con la acreditación, pero también forma parte de ella. Conocer el proceso permite identificar lo que implica obtener una acreditación, así como el análisis de las debilidades y fortalezas con las que cuentan los programas y que parte importante de identificarlas es poder trabajar en ellas de una forma ordenada y constante, lo cual deriva en una mejora continua.

\section{Referencias}

ACBSP. (2013). ACBS SEARCH. Obtenido de https://www.acbspsearch.org/

ACBSP. (s.f.). Who we are. Obtenido de https://www.acbsp.org/page/about-who-we-are

Audina J. S. (2003). La dimensión internacional en los procesos de evaluación y acreditación de la educación superior. Recuperado de http://www. cna. gov. co/1741/articles186502_doc_academico9.pdf.

CHEA. (s.f.). About CIQG. Obtenido de https://www.chea.org/about-ciqg

CHEA. (s.f.). Directories. Obtenido de https://www.chea.org/directories

COPAES. (junio de 2019). ¿Qué es la acreditación? Obtenido de www.copaes.org/acreditacion.php

Cruz López, Y., \& Cruz López, A. K. (2008). La educación superior en México tendencias y desafíos. Avaliação: Revista da Avaliação da Educação Superior (Campinas), 13(2), 293-311.

Egido Gálvez, I., \& Haug, G. (2006). La acreditación como mecanismo de garantía de la calidad: tendencias en el Espacio Europeo de Educación Superior.
Iglesias, D. M. (2004). Generalidades sobre la Metodología de la Investigación. En M. E. Cortés Cortés, \& M. Iglesias León. Ciudad del Carmen, Campeche, México.

INQAAHE. (s.f.). About Interntional Network for Quality Assurance Agencies in Higher Education. Obtenido de https://www.inqaahe.org/

Medina, E. V. C., \& Valdez, E. O. (2011). El proceso de acreditación en programas de Educación Superior: un estudio de caso. Omnia, 17(2), 53-70.

Salas Durazo, Iván Alejandro. (2013). La acreditación de la calidad educativa y la percepción de su impacto en la gestión académica: el caso de una institución del sector no universirario en México. Calidad en la educación, (38), 305-333.

Silas Casillas, J. C. (2014). Calidad y acreditación en la educación superior: realidades y retos para América Latina. Páginas de educación, 7(2), 104-123.

World wide learn. (s.f.). What is an accreditation? Obtenido de https://www.worldwidelearn.com/accreditation/ index.html 